\title{
Celecoxib upregulates ULBP-1 expression in lung cancer cells via the JNK/PI3K signaling pathway and increases susceptibility to natural killer cell cytotoxicity
}

\author{
JAYOUNG KIM ${ }^{1}$, MIN HYE NOH ${ }^{2}$, DAE YOUNG HUR ${ }^{2}$, \\ BOMI KIM ${ }^{3}$, YEONG SEOK KIM ${ }^{2}$ and HYUN-KYUNG LEE ${ }^{4}$
}

${ }^{1}$ Department of Medical Science, Pusan National University School of Medicine, Yangsan, South Gyeongsang 50612; ${ }^{2}$ Department of Anatomy, Inje University College of Medicine, Busan 47396; ${ }^{3}$ Department of Pathology, Inje University Haewoondae Paik Hospital, Busan 48108; ${ }^{4}$ Division of Pulmonology, Department of Internal Medicine, Inje University Pusan Paik Hospital, Busan 47396, Republic of Korea

Received February 20, 2020; Accepted September 8, 2020

DOI: $10.3892 / \mathrm{ol} .2020 .12142$

\begin{abstract}
Lung cancer has the highest cancer mortality rate in the world, and effective therapies are still required. Cyclooxygenase-2 (COX-2) is highly expressed in numerous types of cancer, and is therefore considered a possible target of cancer treatment. Celecoxib, a selective COX-2 inhibitor, has binding pockets that interact with COX-2 and disrupt its enzymatic activities. In addition, celecoxib is able to affect cellular functions in a COX-2-independent manner. The present study aimed to investigate if celecoxib affected natural killer (NK) cell receptors and susceptibility to NK cell toxicity. For this purpose, PCR, immunoblotting, flow cytometry analysis and NK cell cytotoxicity assays were performed. The present study revealed that sublethal concentrations of celecoxib increased the expression levels of UL16-binding protein 1 (ULBP-1), a natural-killer group 2 member D (NKG2D) ligand, in lung cancer A549 and H460 cell lines. ULBP-1 mRNA and protein expression was induced in a dose- and time-dependent manner after celecoxib treatment. Expression levels of other NKG2D ligands, such as ULBP-2, ULBP-3, MHC class I-related chain A (MICA) and MICB did not change considerably compared to ULBP-1 in response to celecoxib treatment. Fluorescence microscopic
\end{abstract}

Correspondence to: Professor Hyun-Kyung Lee, Division of Pulmonology, Department of Internal Medicine, Inje University Pusan Paik Hospital, 75 Bokji-ro, Busanjin-gu, Busan 47396, Republic of Korea

E-mail: goodoc@gmail.com

Professor Yeong Seok Kim, Department of Anatomy, Inje University College of Medicine, 75 Bokji-ro, Busanjin-gu, Busan 47396, Republic of Korea

E-mail: newsoft@inje.ac.kr

Key words: cyclooxygenase-2, celecoxib, UL16-binding protein 1, lung cancer images revealed abundant ULBP-1 in the cytoplasm after celecoxib treatment. Both JNK and PI3K may be involved in the induction of ULBP-1 expression after celecoxib treatment in A549 and H460 cells. In a NK cytotoxicity assay, celecoxib increased the sensitivity to NK cell-mediated cytotoxicity via interaction with ULBP-1 in lung cancer cells. Overall, the present results demonstrated that celecoxib treatment induced ULBP-1 expression in lung cancer cells, thereby increasing their susceptibility to NK cell cytotoxicity. These results suggest that the effects of conventional anticancer therapy may potentially be enhanced by using celecoxib, which targets COX-2, to enhance the sensitivity of lung cancer cells to NK cell-mediated cytotoxicity.

\section{Introduction}

Lung cancer is one of the most common types (2.09 million cases; $11.6 \%$ among cancers; 2018) of cancer in the world, and lung cancer-associated mortality (1.76 million deaths; $18.4 \%$ of total cancer deaths; 2018) is higher than that of other types of cancer (1-3). Only patients with early stage lung cancer who undergo surgery have a reasonable chance of survival; however, recurrence rates of lung cancer are high (2). In addition to conventional chemotherapy, alternative therapy (4) or targeted therapy, such as therapies targeting EGFR, VEGF or cyclooxygenase (COX), have been investigated (5-8). $\mathrm{COX}$ is an essential enzyme to produce prostaglandins and it is also involved in numerous cellular responses, such as cell growth, survival and regulation of proteins $(9,10)$. COX can be inhibited by non-steroidal anti-inflammatory drugs (NSAIDs), which are widely used to control pain, to suppress inflammation and for cancer treatment (8). There are two types of COX enzymes, namely COX-1, and COX-2. The use of NSAIDs for prolonged periods of time can result in side effects, such as intestinal bleeding or gastric ulcer due to inhibition of COX-1 (11). However, inhibition of COX-2 has few side effects, and therefore COX-2 inhibitors are widely used to relieve pain and to treat inflammatory diseases including rheumatoid arthritis and osteoarthritis (12). 
Celecoxib is a selective COX-2 inhibitor with little effect on COX-1 (12). Celecoxib has been reported to have anticancer effects in colon $(13,14)$, gastric $(15,16)$, breast $(17)$, lung $(18,19)$ and prostate $(20,21)$ cancer. Celecoxib inhibits tumor growth, induces apoptosis and cell cycle arrest of cancer cells, and suppresses tumor angiogenesis $(11,22,23)$. Therefore, celecoxib has been investigated as an adjuvant agent in cancer therapeutics. Clinicians have tried to use celecoxib as an adjuvant agent to treat colorectal, lung, melanoma and breast cancer, but it is not standard treatment yet (24).

The natural-killer group 2 member D (NKG2D) receptor is a C-type lectin-like activating receptor $(25,26)$. NKG2D is a homodimeric receptor expressed by cytotoxic lymphocytes and encoded by the natural killer (NK) cell gene complex (26). NKG2D is expressed by several types of cells, including NK, CD8 $\alpha \beta \mathrm{T}, \gamma \delta \mathrm{T}$, NKT and a small subset of CD4 $\alpha \beta$ $\mathrm{T}$ cells $(25,26)$. Therefore, NKG2D recognition is considered to be critical in tumor immune surveillance. NKG2D binds to MHC class I-related chain A (MICA), MICB and UL16-binding proteins 1-6 (ULBP-1-6) in humans (25-27). NKG2D ligands are expressed on various cancer cells, but are rarely expressed on healthy cells (28). Expression of NKG2D ligands can induce heat shock stress, DNA damage and post-transcriptional epigenetic modifications in cancer cells (28). Cancer therapies that increase NKG2D ligand expression may therefore have a therapeutic effect. The present study investigated the induction of ULBP-1 expression in lung cancer cells after celecoxib treatment and explored the susceptibility of these celecoxib-treated cells to NK cell cytotoxicity.

\section{Materials and methods}

Cell culture. The human lung cancer A549 and H460 cell lines were purchased from the American Type Culture Collection (ATCC) and were maintained in RPMI-1640 medium supplemented with $10 \% \mathrm{FBS}, 100 \mathrm{U} / \mathrm{ml}$ penicillin and $100 \mu \mathrm{g} / \mathrm{ml}$ streptomycin (all HyClone; Cytiva). The human NK NK-92MI cell line (ATCC) was maintained in $\alpha$ Minimum Essential Medium (HyClone; Cytiva) supplemented with $2 \mathrm{mM}$ L-glutamine, $0.2 \mathrm{mM}$ inositol, $20 \mathrm{mM}$ folic acid and $15 \%$ FBS. Cells were grown in a humidified incubator at $37^{\circ} \mathrm{C}$ with $5 \% \mathrm{CO}_{2}$. NK-92MI cells were maintained with optimal cell density between $2 \times 10^{5}$ and $8 \times 10^{5}$ cells $/ \mathrm{ml}$. A549 and H450 cells were maintained between $5 \times 10^{3}$ and $5 \times 10^{4}$ cells $/ \mathrm{cm}^{2}$.

Celecoxib and chemical treatment. A549 or H460 cells $\left(1 \times 10^{4}\right.$ cells $\left./ \mathrm{cm}^{2}\right)$ were initially placed in a culture dish. After $8 \mathrm{~h}$ of incubation at $37^{\circ} \mathrm{C}$, the attached cells were treated with various concentrations $(0,25,50,75,100$ and $200 \mu \mathrm{M})$ of celecoxib (Toronto Research Chemicals) at $37^{\circ} \mathrm{C}$ overnight. To examine time-dependent increase of ULBP-1, A549 and H460 cells were treated with $75 \mu \mathrm{M}$ celecoxib for $2,4,6$, $8,10,12$ and $24 \mathrm{~h}$. To investigate the expression levels of NKG2D ligands, cells were treated with sublethal concentrations (50 or $75 \mu \mathrm{M}$ ) of celecoxib. For inhibition of PI3K or JNK pathway, cells were preincubated with $10 \mu \mathrm{M}$ LY294002 (EMD Millipore), a PI3K inhibitor, or $10 \mu \mathrm{M}$ SP600125 (EMD Millipore), a JNK inhibitor, for $1 \mathrm{~h}$ at $37^{\circ} \mathrm{C}$ before celecoxib treatment. Cells were further treated with celecoxib as aforementioned without removing the medium containing the inhibitors.

Analysis of cytotoxicity. Cell viability was measured using the WST-1 assay (Takara Bio, Inc.) according to the manufacturer's protocol. A549 and H460 cells (5x10\%3/well) were seeded in 96 -well plates and incubated at $37^{\circ} \mathrm{C}$ for $24 \mathrm{~h}$. Cells were treated with various concentrations of celecoxib overnight, as aforementioned. The next day, $10 \mu \mathrm{l}$ of WST-1 was added to each well, and plates were incubated at $37^{\circ} \mathrm{C}$ for $1 \mathrm{~h}$. Absorbance was measured using a microplate reader (Pierce; Thermo Fisher Scientific, Inc.) at a wavelength of $450 \mathrm{~nm}$.

RNA isolation and reverse transcription (RT)-PCR. Total RNA was extracted from A549 and H460 cells using TRIzol ${ }^{\circledR}$ reagent (Invitrogen; Thermo Fisher Scientific, Inc.) according to the manufacturer's protocol and was used to synthesize cDNA at $42^{\circ} \mathrm{C}$ for $1 \mathrm{~h}$ using oligo dT primers and AccuPower ${ }^{\circledR}$ RT-premix (Bioneer Corporation). cDNA was amplified using Tenuto PCR premix (Enzynomics Co., Ltd.) and a PCR thermal cycler (Takara Bio, Inc.) with the following thermocycling conditions: $5 \mathrm{~min}$ at $95^{\circ} \mathrm{C}$ (pre-denaturation), 30 cycles of $20 \mathrm{sec}$ at $94^{\circ} \mathrm{C}, 10 \mathrm{sec}$ at $55^{\circ} \mathrm{C}$ (for MICA/B) or $65^{\circ} \mathrm{C}$ (for ULBPs), $30 \mathrm{sec}$ at $72^{\circ} \mathrm{C}$ and $5 \mathrm{~min}$ at $72^{\circ} \mathrm{C}$ (final extension). The following primer pairs were used: ULBP-1 forward, 5'-GCCAGGATGTCTTGTGAGCA-3' and reverse, 5'-CAG TGGTGAGTAGACAGGCG-3'; ULBP-2 forward, 5'-CCC TGGGGAAGAAACTAAATGTC-3' and reverse, 5'-ACT GAACTGCCAAGATCCACTGCT-3'; ULBP-3 forward, 5'-GAGGCTCAGACTGGAACTGG-3' and reverse, 5'-GCC TCTTCTTCCTGTGCATC-3'; MICA forward, 5'-CAGACT GCCTGCAGGAACTA-3' and reverse, 5'-TTTCTTCTTACA ACAACGGACATA-3'; MICB forward, 5'-CGGACAGAC TTTCCATAT GTTT-3' and reverse, 5'-TCCAACAACAAT AAATAAGTG ATG-3'; and $\beta$-actin forward, 5'-CATCGT GATGGACTCCGGTGAC-3' and reverse, 5'-TCAGGT AGTCAGTCAGGTCC-3'. The PCR products were analyzed via $1 \%$ agarose gel electrophoresis in TAE buffer with ethidium bromide and a UV illuminator. The densities of the bands were measured using ImageJ v1.53 software (National Institutes of Health).

Western blot analysis. Whole cell lysates from A549 and H460 cells (4x105/60-mm culture dish) were prepared in RIPA lysis buffer (Invitrogen; Thermo Fisher Scientific, Inc.) supplemented with a protease inhibitor cocktail (Sigma-Aldrich; Merck KGaA). Total protein in the cell lysates was determined using the BCA assay, and sample proteins (10 $\mu \mathrm{g} /$ lane) were separated via $10 \%$ SDS-PAGE. Separated proteins were transferred onto nitrocellulose membranes after separation by electrophoresis. Membranes were blocked with 5\% skimmed milk at room temperature for $2 \mathrm{~h}$ and washed three times with TBS-0.1\% Tween (TBST). Primary antibodies against ULBP-1 (1:1,000; cat. no. ab176566, Abcam) and $\beta$-actin (1:2,000; cat. no. sc-69879, Santa Cruz Biotechnology, Inc.) were used. Following incubation with primary antibodies in TBST at $4^{\circ} \mathrm{C}$ overnight, unbound antibodies were washed away with TBST. Bound primary antibodies were visualized via incubation of blots with HRP-conjugated anti-rabbit secondary antibodies (1:5,000; cat. no. 7074; Cell Signaling 
A
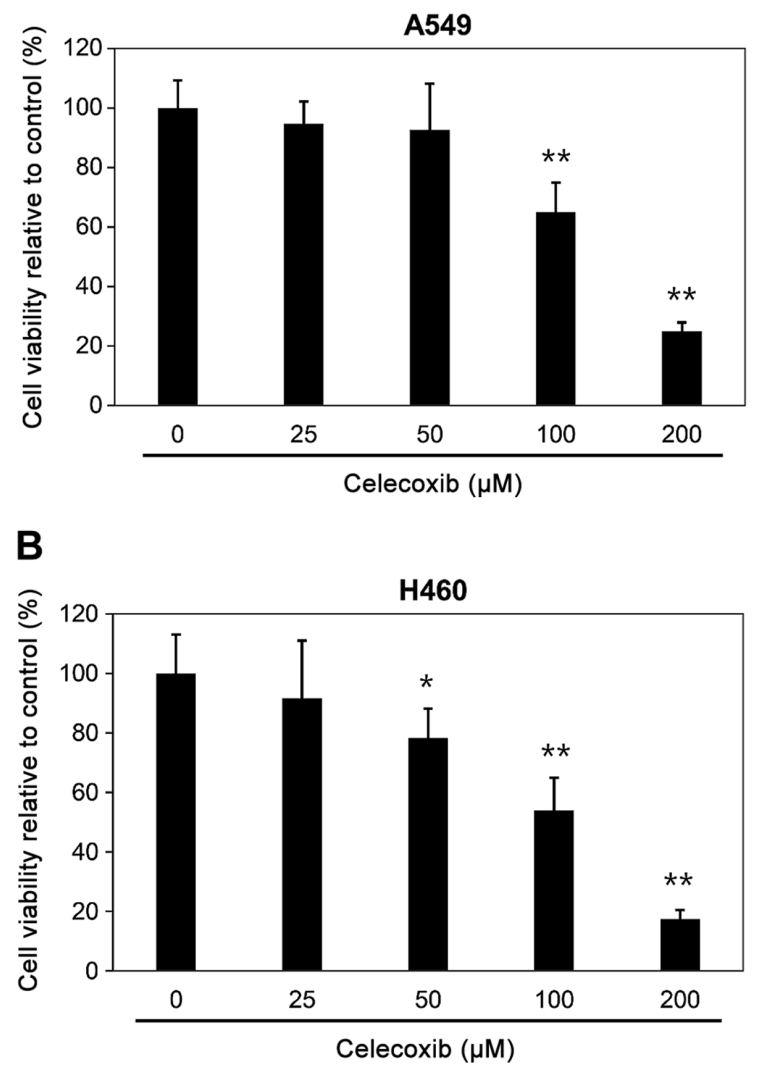

Figure 1. Cell viability of lung cancer cells after treatment with various concentrations of celecoxib. (A) A549 and (B) H460 cells were treated with various concentrations of celecoxib $(0,25,50,100$ and $200 \mu \mathrm{M})$ overnight. Cell viability was measured using a WST-1 assay. Graphs present the mean \pm standard deviation from three separate experiments performed in triplicate. Inhibition of each celecoxib-treated group relative to control group was evaluated using a one-way ANOVA with Bonferroni correction. ${ }^{* *} \mathrm{P}<0.005 ;{ }^{*} \mathrm{P}<0.05$.

Technology, Inc.) or HRP-conjugated anti-mouse secondary antibodies (1:5,000; cat. no. 7076; Cell Signaling Technology, Inc.) at room temperature for $1 \mathrm{~h}$, and the signal was detected using chemiluminescence detection reagents (Amersham; Cytiva). The densities of bands were measured using ImageJ software version 1.53 (National Institutes of Health).

Flow cytometry analysis and fluorescence microscopy analysis of ULBP-1 expression. For flow cytometry, $1 \times 10^{6}$ cells of A549 and H460 were incubated with anti-ULBP-1 antibody (1 $\mu \mathrm{g} / \mathrm{ml}$; rabbit IgG; cat. no. ab176566, Abcam) in PBS containing $0.1 \%$ FBS for $30 \mathrm{~min}$ on ice. Cells were washed and incubated with Alexa flour 488-conjugated secondary antibody for $30 \mathrm{~min}$ on ice (1:1,000; cat. no. AP132JA4; Sigma-Aldrich; Merck KGaA). Labeled cells were washed twice and resuspended in PBS. Flow cytometry data were obtained using a MACSquant flow cytometer (Miltenyi Biotec $\mathrm{GmbH}$ ) and analyzed by MACSquant analysis software version 2.6.1517 (Miltenyi Biotech, GmbH) embedded in the machine. For fluorescence microscopy, labeled cells were collected onto glass slides using a Cytospin centrifuge (Hanil Science Industrial Co., Ltd.). Cells were analyzed via fluorescence microscopy (magnification, x20) using the iRiS digital cell imaging system (Logos Biosystems, Inc.).
A
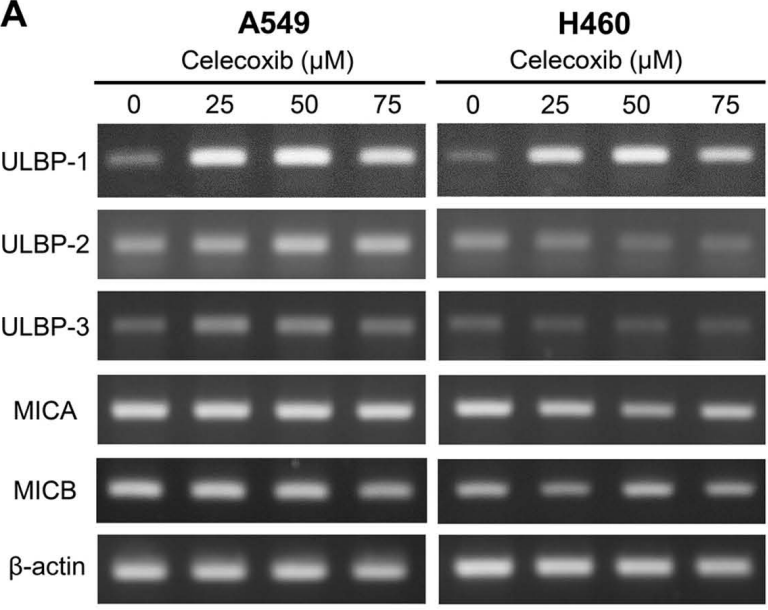

B
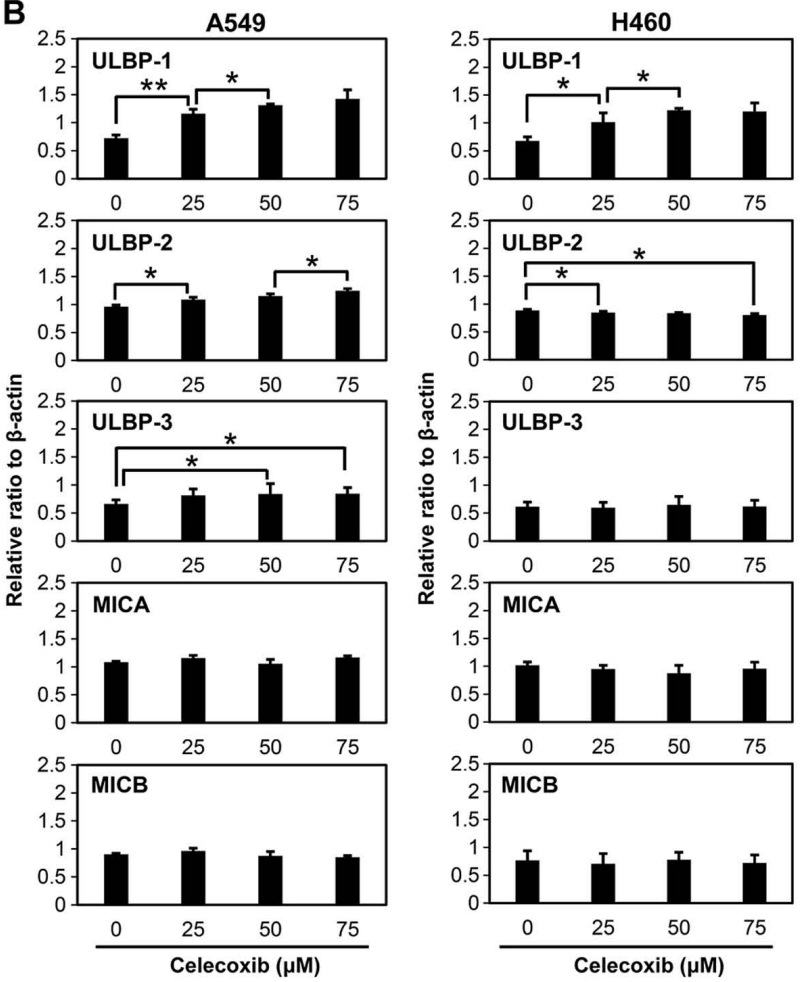

Figure 2. Expression levels of natural-killer group 2 member D ligands in lung cancer cells after celecoxib treatment. A549 and H460 cells were treated with celecoxib $(0,25,50$ and $75 \mu \mathrm{M})$ overnight. ULBP-1/2/3, MICA and MICB mRNA levels were measured using reverse tran-scription-PCR. (A) Representative bands of five independent experiments. (B) Graphs displaying the mean \pm standard deviation of the ratio between densities of indicated mRNAs and $\beta$-actin. Data were analyzed using a one-way ANOVA with Bonferroni correction. ${ }^{* *} \mathrm{P}<0.005 ;{ }^{*} \mathrm{P}<0.05$. MICA/B, MHC class I-related chain A/B; ULBP-1/2/3, UL16-binding protein 1/2/3.

NK cytotoxicity assay. NK cell cytotoxic activity was determined using a calcein-AM assay. Briefly, target cells (A549 and H460) were washed twice with PBS and incubated with 5 mM calcein-AM (Sigma-Aldrich; Merck KGaA) in serum-free RPMI medium for $10 \mathrm{~min}$ at $37^{\circ} \mathrm{C}$. Cells were then treated with/without celecoxib, as aforementioned. Labeled target cells were distributed into the wells of U-bottom microtiter plates at a concentration of $1 \times 10^{4}$ cells/well. NK-92MI cells were used as effector cells and were added at various effector:target (E:T) ratios $(0: 1,2.5: 1,5: 1$ and 10:1) in quadruplicate. Target cells in 
A

A549

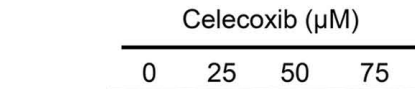

ULBP-1

$\begin{array}{llll}0 & 25 & 50 & 75\end{array}$
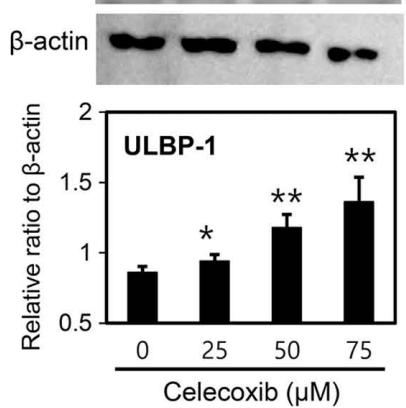

B

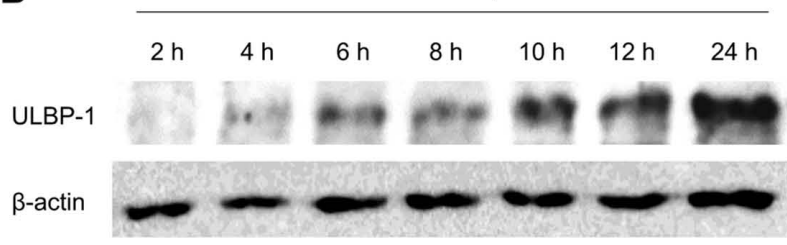

Celecoxib $75 \mu \mathrm{M}$

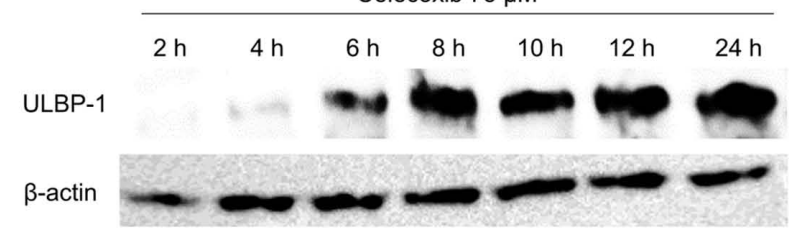

$\mathrm{H} 460$

Celecoxib $(\mu \mathrm{M})$

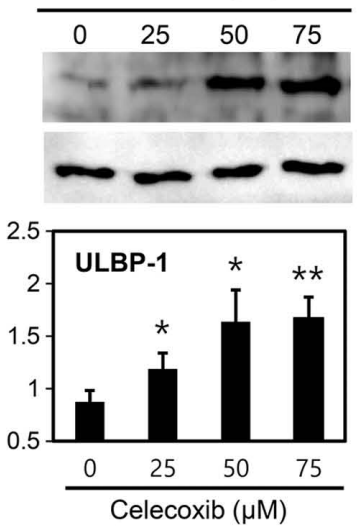

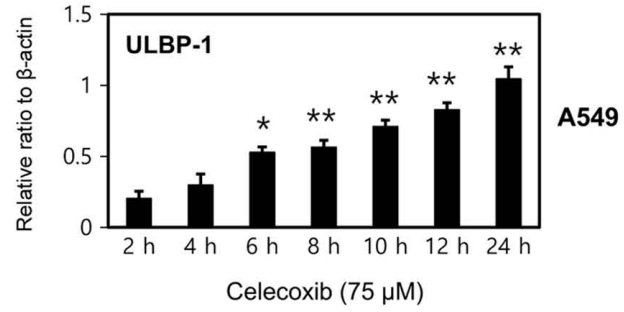

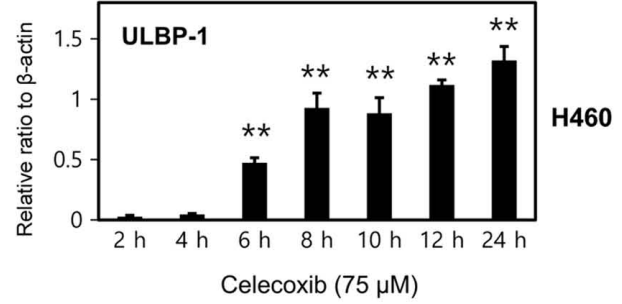

Figure 3. Celecoxib induces ULBP-1 expression in lung cancer cells in a dose- and time-dependent manner. (A) A549 and H460 cells were treated with celecoxib $(0,25,50$ and $75 \mu \mathrm{M})$ overnight. ULBP-1 protein expression was measured via western blot analysis. The blot images shown are representative of seven independent experiments. Graphs showed the mean \pm standard deviation of each relative ratio to $\beta$-actin. (B) A549 and H460 cells were treated with $75 \mu \mathrm{M}$ celecoxib for 2, 4, 6, 8, 10, 12 and $24 \mathrm{~h}$. ULBP-1 protein expression was measured via western blot analysis. The blot images are representative of five independent experiments. Graphs showed the mean \pm standard deviation of each relative ratio to $\beta$-actin. Relative expression levels of ULBP-1 to $\beta$-actin were analyzed using one-way ANOVA with Bonferroni correction. ${ }^{* *} \mathrm{P}<0.005 ;{ }^{*} \mathrm{P}<0.05$. ULBP-1, UL16-binding protein 1.

complete RPMI medium alone were used to determine spontaneous calcein-AM retention. Maximal lysis was determined by solubilizing three wells of target cells in lysis buffer $(0.1 \%$ Triton X-100; Sigma-Aldrich; Merck KGaA). After incubation at $37^{\circ} \mathrm{C}$ for $8 \mathrm{~h}$, assays were analyzed with 490/520 nm (excitement/emission) using a fluorescence reader. Percent specific cytotoxicity was calculated as calcein release relative to maximal lysis.

Statistical analysis. Results are expressed as the mean \pm standard deviation. Statistical analysis was performed using GraphPad Prism 5.01 (GraphPad Software, Inc.). For comparisons among $>2$ groups, data were analyzed using a one-way ANOVA with Bonferroni correction to compare each two pairs between the indicated concentrations and control. In the NK cytotoxicity assay, Student's unpaired two-sided t-test was used to compare the lysis percentage between control and each celecoxib-treated group, or a one-way ANOVA followed by the post hoc Bonferonni test was used to compare multiple groups. $\mathrm{P}<0.05$ was considered to indicate a statistically significant difference.

\section{Results}

Cytotoxicity of celecoxib towards lung cancer cells. Cell viability was determined using a WST-1 assay after celecoxib treatment of the lung cancer A549 and H460 cell lines. Lung cancer cells were incubated with various concentrations of celecoxib $(0,25,50,100$ and $200 \mu \mathrm{M})$ overnight. Cell viabilities of A549 or H460 cells were significantly decreased when treated with $\geq 100 \mu \mathrm{M}$ celecoxib; therefore, $50 \mu \mathrm{M}$ of celecoxib, which exhibited minimal cell cytotoxicity, were the concentrations used in subsequent experiments (Fig. 1).

Expression levels of NKG2D ligands after celecoxib treatment. Expression levels of NKG2D ligands, including ULBP-1, ULBP-2, ULBP-3, MICA and MICB, were examined after treatment of A549 and H460 cells with celecoxib 
A

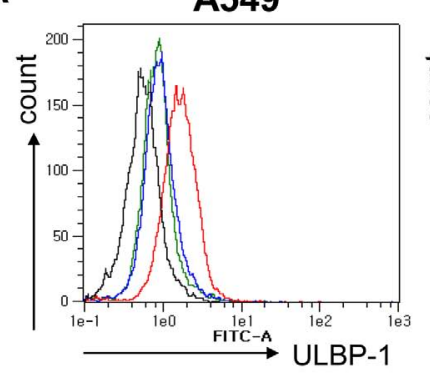

B
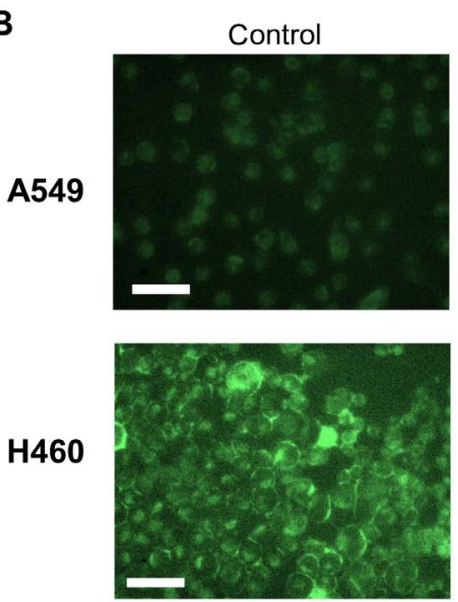

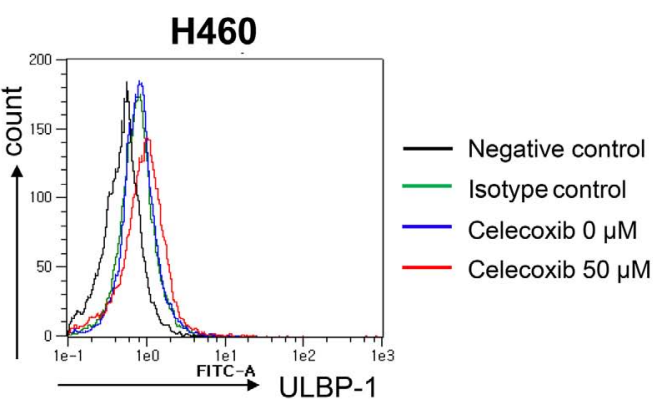

Celecoxib $50 \mu \mathrm{M}$
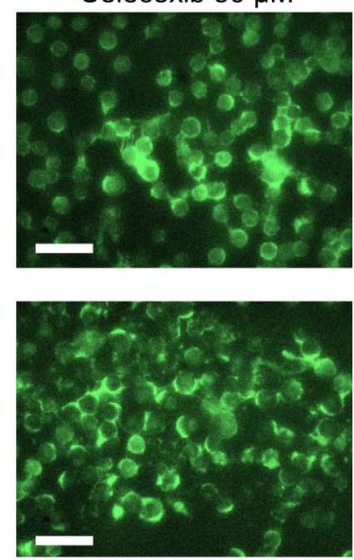

Figure 4. ULBP-1 expression in celecoxib-treated lung cancer cells analyzed via flow cytometry and fluorescence microscopy. A549 and H460 cells were treated with celecoxib $(50 \mu \mathrm{M})$ overnight. (A) ULBP-1 expression on the cell surface was measured via flow cytometry analysis. The data shown are representative of six independent experiments. (B) ULBP-1 was stained using an anti-ULBP-1 antibody and Alexa488-conjugated secondary antibodies, collected using a Cytospin flow cytometer and observed using a fluorescence microscope. Scale bar, $100 \mu \mathrm{m}$. The figures shown are representative of the results from four independent experiments. ULBP-1, UL16-binding protein 1.

(Fig. 2A). ULBP-1 mRNA expression increased when A549 and $\mathrm{H} 460$ cells were treated with 25,50 and $75 \mu \mathrm{M}$ of celecoxib. ULBP-2 expression significantly increased in A549 cells treated with celecoxib compared with the control. In contrast, ULBP-2 at $75 \mu \mathrm{M}$ of celecoxib treatment slightly decreased in H460 cells compared with the control, but this was not statistically significant (Fig. 2B). Celecoxib treatment also increased ULBP-3 in A549 cells compared to the control, however, this was not observed in H460 cells. Transcript levels of other NKG2D ligands, namely MICA and MICB, did not significantly change after celecoxib treatment of A549 and H460 cells (Fig. 2B). Protein expression of ULBP-1 was further investigated as its mRNA expression distinctly. Other ULBPs proteins were also investigated and ULBP-2 protein was also increased on celecoxib-treated A549 cells (data not shown), but the main focus was on ULBP-1 which demonstrated distinct differences. It was further observed that the celecoxib-induced increase in ULBP-1 expression was dose- (Fig. 3A) and time-dependent (Fig. 3B). ULBP-1 expression was significantly increased in both A549 and $\mathrm{H} 460$ cells treated with $\geq 25 \mu \mathrm{M}$ celecoxib (Fig. 3A). Additionally, celecoxib significantly increased ULBP-1 protein expression after $6 \mathrm{~h}$ of treatment (Fig. 3B). The increase in ULBP-1 expression was also investigated via flow cytometry analysis (Fig. 4A) and fluorescence microscopy (Fig. 4B). ULBP-1 was more strongly expressed by A549 than H460 cells after celecoxib treatment (Fig. 4A). Fluorescence microscopic images revealed abundant ULBP-1 in the cytoplasm after celecoxib treatment (Fig. 4B).

Association between ULBP-1 expression and JNK and PI3K signaling pathways. Cells were preincubated for $1 \mathrm{~h}$ with LY294002 $(10 \mu \mathrm{M})$ as a PI3K inhibitor or SP600125 $(10 \mu \mathrm{M})$ as a JNK inhibitor, and were then treated with a sublethal concentration $(50 \mu \mathrm{M})$ of celecoxib. ULBP-1 expression was determined via western blotting. SP600125 significantly attenuated the induction of ULBP-1 expression after celecoxib treatment of A549 and H460 cells ( $\mathrm{P}<0.05$; Fig. 5A). Additionally, LY294002 significantly attenuated ULBP-1 induction in A549 $(\mathrm{P}<0.05)$, but not $\mathrm{H} 460$ cells $(\mathrm{P}=0.071)$ (Fig. 5B).

Susceptibility of lung cancer cells to NK cell-mediated lysis. Finally, the present study investigated whether the celecoxib-induced increase in ULBP-1 expression resulted in increased NK cell-mediated lysis. Celecoxib-treated A549 and H460 cells were co-cultured with various ratios of NK-92MI cells, and then NK cell toxicity was measured using a calcein assay. NK-91MI cells were significantly cytotoxic to celecoxib-treated A549 cells at 5:1 and 10:1 E:T ratios, while NK cell-mediated toxicity of celecoxib-treated H460 cells was only observed at a 10:1 E:T ratio (Fig. 6A). Both SP600125 and LY294002 significantly attenuated NK cell susceptibility of A549 cells after celecoxib treatment $(\mathrm{P}<0.05$; Fig. 6B). 
A
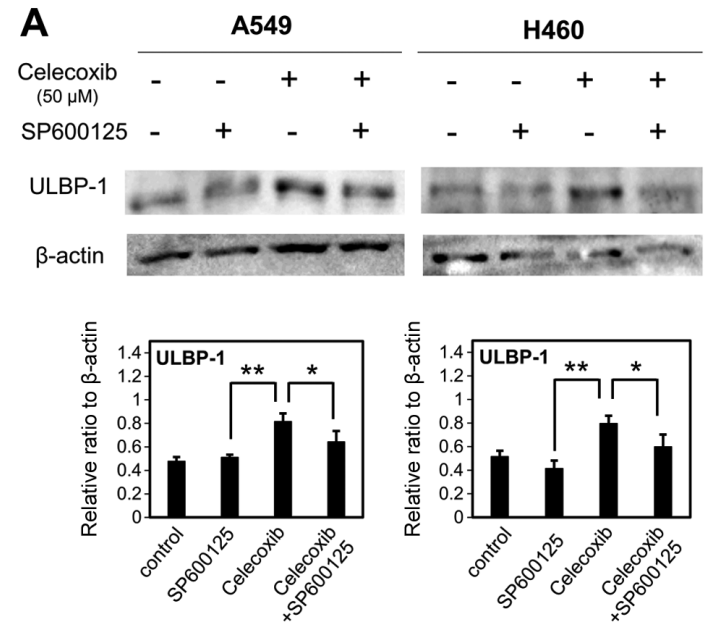

B
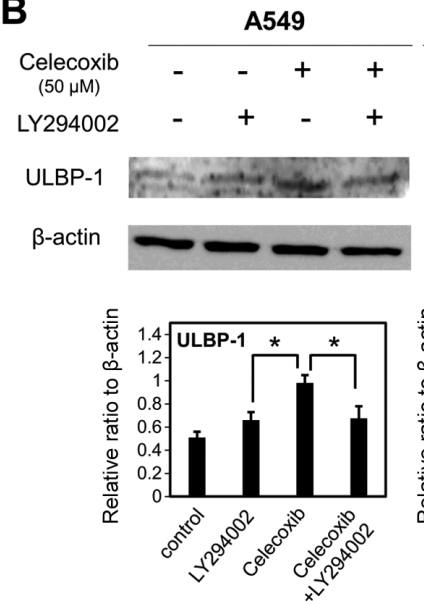
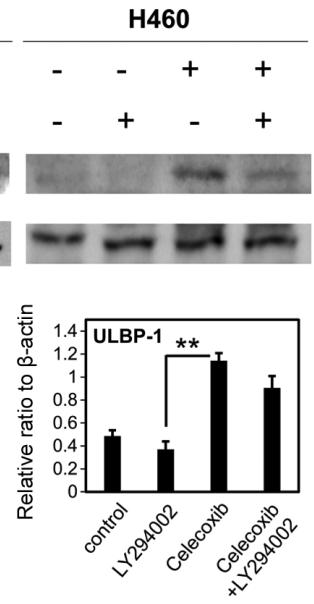

Figure 5. Association between ULBP-1 expression and JNK/PI3K in celecoxib-treated lung cancer cells. A549 and H460 cells were treated with $50 \mu \mathrm{M}$ celecoxib overnight after pretreatment with SP600125 and LY294002 for $1 \mathrm{~h}$. (A) Protein expression levels of ULBP-1 after SP600125 pretreatment of A549 and H460 measured via western blotting. Data shown are based on three independent experiments. (B) Protein expression levels of ULBP-1 after LY294002 pretreatment of A549 and H460 measured via western blotting. The blot images shown are representative of five independent experiments. Graphs showed the mean \pm standard deviation of each relative ratio to $\beta$-actin. Relative expression levels of ULBP-1 to $\beta$-actin were analyzed using one-way ANOVA with Bonferroni correction. ${ }^{* *} \mathrm{P}<0.005 ;{ }^{*} \mathrm{P}<0.05$. ULBP-1, UL16-binding protein 1.

A

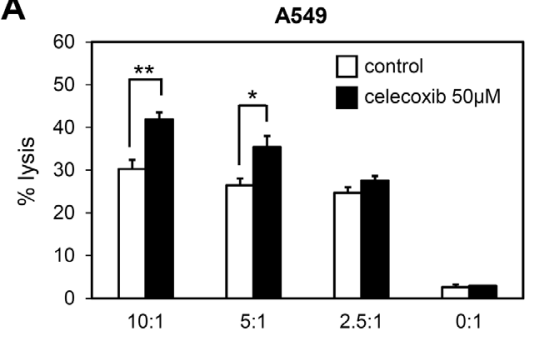

B

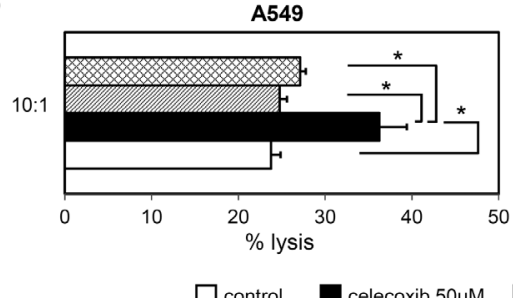

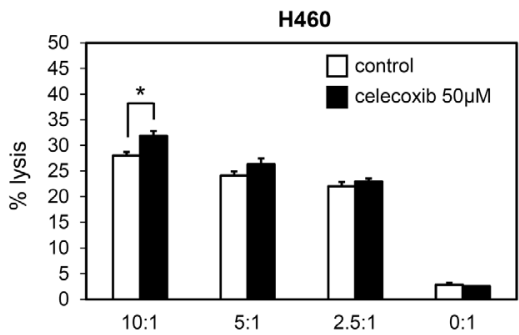

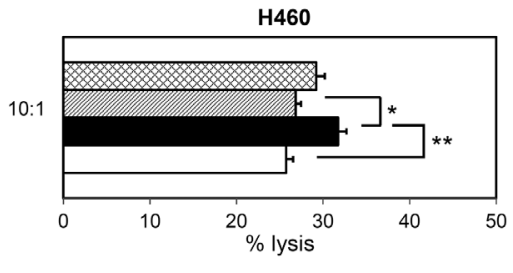

celecoxib 50

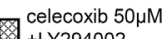

Figure 6. NK cell cytotoxicity towards celecoxib-treated lung cancer cells. (A) A549 and H460 cells were stained with calcein-AM and treated with celecoxib $(50 \mu \mathrm{M})$ overnight. (B) SP600125 or LY294002 were used for pretreatment before celecoxib treatment. Target cells (1x10 ${ }^{4}$ cells) were incubated with various effector:target ratios (10:1, 5:1, 2.5:1 and 0:1) of NK-92MI cells as effector cells for $8 \mathrm{~h}$. Data are shown as the mean \pm standard deviation of three independent experiments. To compare the \% lysis between control and celecoxib-treated groups (A), unpaired two-sided Student's t-tests were used. To compare multiple groups in (B), a one-way ANOVA with Bonferroni correction was used. ${ }^{* *} \mathrm{P}<0.005 ;{ }^{*} \mathrm{P}<0.05$. NK, natural killer.

Additionally, SP600125, but not LY249002, significantly attenuated NK cell-mediated toxicity of celecoxib-treated $\mathrm{H} 460$ cells $(\mathrm{P}<0.05$ and $\mathrm{P}=0.060$, respectively; Fig. 6B).

\section{Discussion}

Celecoxib was developed as a COX-2 selective inhibitor, which is a type of NSAID that is used as an analgesic. COX-2 is highly expressed in numerous types of cancer, such as colorectal cancer, breast cancer and non-small cell lung cancer, and is therefore a possible target of cancer therapy (7). By targeting COX-2, celecoxib has attracted attention as an adjuvant therapeutic drug for cancer treatment (8). However, it has been reported that celecoxib can regulate intracellular functions (autophagy or ER stress), and cell signaling (PI3K or MAPK), as well as inhibit COX-2 function (16-23). In the current study, an increase in transcript and protein expression levels of ULBP-1, an activating receptor for NK cells, was observed in lung cancer cells treated with celecoxib.

The increase in ULBP-1 expression was the most prominent compared with other NKG2D, such as ULBP-2, ULBP-3, MICA or MICB. Celecoxib was able to induce both mRNA and protein expression levels of ULBP-1 in A549 and H460 cells in a dose- and time-dependent manner. Kim et al (29) reported that celecoxib induced ULBP-1 expression in colon cancer cells in a COX-2 independent manner. The present study revealed that not only ULBP-2 expression was increased 
by treatment of A549 and H460 cells with celecoxib, but also ULBP-3 expression was increased on A549 cells following celecoxib treatment. ULBP-3 on H460 was not significantly changed. MICA and MICB expression, on the other hand, was not affected by celecoxib treatment. It was concluded that activating NKG2D ligands (ULBPs) were more highly expressed by celecoxib-treated lung cancer cells than inhibitory NKG2D ligands (MICA/B), as celecoxib-treated lung cancer cells were susceptible to NK cell-mediated death. However, interactions between NKG2D ligands and celecoxib treatment should be studied further in other lung cancer cells that express various types of EGFR and KRAS mutations (30), because both A549 and H460 have wild-type EGFR.

Extrinsic stimuli, such as stress and drugs, can activate the MAPK and PI3K signaling pathways (31). The MAPK signaling pathway was reported as a regulator of NKG2D ligand expression, including ULBPs (32). The PI3K signaling pathway is also involved in NKG2D ligand regulation (33). Therefore, since celecoxib may produce cell stress and modulate the MAPK or PI3K signaling pathways (31-33), it may be involved in the regulation of NKG2D ligands. In the present study, SP600125 (a JNK inhibitor) and LY294002 (a PI3K inhibitor) decreased ULBP-1 expression in celecoxib-treated lung cancer cells. However, the present study did not investigate whether celecoxib may directly regulate the PI3K or JNK signaling pathways. It is possible that other mediators affected by JNK or PI3K may be associated with celecoxib-mediated ULBP-1 expression. More precise experiments are required to investigate this further.

Overall, the present results demonstrated that treatment of lung cancer cells with a sublethal concentration of celecoxib induced ULBP-1 expression without cell toxicity, and increased the susceptibility of these cancer cells to NK cell cytotoxicity. The current results indicated that celecoxib may potentially increase the effects of conventional anticancer therapy by making lung cancer cells more sensitive to NK cells, in addition to targeting COX-2.

\section{Acknowledgements}

Not applicable.

\section{Funding}

The present study was supported by a 2016 research grant from Inje University Busan Paik Hospital. This grant was an internal research fund provided by the university itself.

\section{Availability of data and materials}

The data used and/or analysed during the current study are available from the corresponding author on reasonable request.

\section{Authors' contributions}

HKL and YSK contributed to conception and design and interpretation of data. JK and MHN contributed to acquisition of data and drafting the manuscript. DYH and BK contributed to interpretation of data. All authors have read and approved the manuscript.

\section{Ethics approval and consent to participate}

Not applicable.

\section{Patient consent for publication}

Not applicable.

\section{Competing interests}

The authors declare that they have no competing interest.

\section{References}

1. Herbst RS, Heymach JV and Lippman SM: Lung cancer. N Engl J Med 359: 1367-1380, 2008.

2. Bade BC and Dela Cruz CS: Lung Cancer 2020: Epidemiology, Etiology, and Prevention. Clin Chest Med 41: 1-24, 2020.

3. Hirsch FR and Lippman SM: Advances in the biology of lung cancer chemoprevention. J Clin Oncol 23: 3186-3197, 2005.

4. Kasymjanova G, Tran AT, Cohen V, Pepe C, Sakr L, Small D, Agulnik JS and Jagoe RT: The use of a standardized Chinese herbal formula in patients with advanced lung cancer: a feasibility study. J Integr Med 16: 390-395, 2018.

5. Dy GK and Adjei AA: Novel targets for lung cancer therapy: Part I. J Clin Oncol 20: 2881-2894, 2002.

6. Singhal S, Vachani A, Antin-Ozerkis D, Kaiser LR and Albelda SM: Prognostic implications of cell cycle, apoptosis, and angiogenesis biomarkers in non-small cell lung cancer: A review. Clin Cancer Res 11: 3974-3986, 2005.

7. Ghosh N, Chaki R, Mandal V and Mandal SC: COX-2 as a target for cancer chemotherapy. Pharmacol Rep 62: 233-244, 2010.7.

8. Gasparini G, Longo R, Sarmiento R and Morabito A: Inhibitors of cyclo-oxygenase 2: A new class of anticancer agents? Lancet Oncol 4: 605-615, 2003

9. Kalinski P: Regulation of immune responses by prostaglandin E2. J Immunol 188: 21-28, 2012.

10. Williams CS, Mann M and DuBois RN: The role of cyclooxygenases in inflammation, cancer, and development. Oncogene 18: 7908-7916, 1999

11. Liggett JL, Zhang X, Eling TE and Baek SJ: Anti-tumor activity of non-steroidal anti-inflammatory drugs: Cyclooxygenaseindependent targets. Cancer Lett 346: 217-224, 2014.

12. Katori $\mathbf{M}$ and Majima M: Cyclooxygenase-2: Its rich diversity of roles and possible application of its selective inhibitors. Inflamm Res 49: 367-392, 2000.

13. Gungor H, Ilhan $\mathrm{N}$ and Eroksuz $\mathrm{H}$ : The effectiveness of cyclooxygenase- 2 inhibitors and evaluation of angiogenesis in the model of experimental colorectal cancer. Biomed Pharmacother 102: 221-229, 2018.

14. Huang S and Sinicrope FA: Celecoxib-induced apoptosis is enhanced by ABT-737 and by inhibition of autophagy in human colorectal cancer cells. Autophagy 6: 256-269, 2010.

15. Liu M, Li CM, Chen ZF, Ji R, Guo QH, Li Q, Zhang HL and Zhou YN: Celecoxib regulates apoptosis and autophagy via the PI3K/Akt signaling pathway in SGC-7901 gastric cancer cells. Int J Mol Med 33: 1451-1458, 2014.

16. Kim N, Kim CH, Ahn DW, Lee KS, Cho SJ, Park JH, Lee MK, Kim JS, Jung HC and Song IS: Anti-gastric cancer effects of celecoxib, a selective COX-2 inhibitor, through inhibition of Akt signaling. J Gastroenterol Hepatol 24: 480-487, 2009.

17. Dai ZJ, Ma XB, Kang HF, Gao J, Min WL, Guan HT, Diao Y, Lu WF and Wang XJ: Antitumor activity of the selective cyclooxygenase- 2 inhibitor, celecoxib, on breast cancer in vitro and in vivo. Cancer Cell Int 12: 53, 2012.

18. Kim B, Kim J and Kim YS: Celecoxib induces cell death on non-small cell lung cancer cells through endoplasmic reticulum stress. Anat Cell Biol 50: 293-300, 2017.

19. Schellhorn M, Haustein M, Frank M, Linnebacher M and Hinz B: Celecoxib increases lung cancer cell lysis by lymphokine-activated killer cells via upregulation of ICAM-1. Oncotarget 6: 39342-39356, 2015.

20. Bieniek J, Childress C, Swatski MD and Yang W: COX-2 inhibitors arrest prostate cancer cell cycle progression by down-regulation of kinetochore/centromere proteins. Prostate 74: 999-1011, 2014. 
21. Katkoori VR, Manne K, Vital-Reyes VS, Rodríguez-Burford C, Shanmugam C, Sthanam M, Manne U, Chatla C, Abdulkadir SA and Grizzle WE: Selective COX-2 inhibitor (celecoxib) decreases cellular growth in prostate cancer cell lines independent of p53. Biotech Histochem 88: 38-46, 2013.

22. Grösch S, Maier TJ, Schiffmann S and Geisslinger G: Cyclooxygenase-2 (COX-2)-independent anticarcinogenic effects of selective COX-2 inhibitors. J Natl Cancer Inst 98: 736-747, 2006.

23. Wu T, Leng J, Han C and Demetris AJ: The cyclooxygenase-2 inhibitor celecoxib blocks phosphorylation of Akt and induces apoptosis in human cholangiocarcinoma cells. Mol Cancer Ther 3: 299-307, 2004.

24. Tołoczko-Iwaniuk N, Dziemiańczyk-Pakieła D, Nowaszewska BK, Celińska-Janowicz K and Miltyk W: Celecoxib in cancer therapy and prevention - review. Curr Drug Targets 20: 302-315, 2019.

25. Bryceson YT and Ljunggren HG: Tumor cell recognition by the NK cell activating receptor NKG2D. Eur J Immunol 38 2957-2961, 2008

26. Waldhauer I and Steinle A: NK cells and cancer immunosurveillance. Oncogene 27: 5932-5943, 2008.

27. Raulet DH: Roles of the NKG2D immunoreceptor and its ligands. Nat Rev Immunol 3: 781-790, 2003.

28. Dhar P and Wu JD: NKG2D and its ligands in cancer. Curr Opin Immunol 51: 55-61, 2018
29. Kim SJ, Ha GH, Bae JH, Kim GR, Son CH, Park YS, Yang K, Oh SO, Kim SH and Kang CD: COX-2- and endoplasmic reticulum stress-independent induction of ULBP-1 and enhancement of sensitivity to NK cell-mediated cytotoxicity by celecoxib in colon cancer cells. Exp Cell Res 330: 451-459, 2015.

30. Reckamp KL: Molecular Targets Beyond the Big 3. Thorac Surg Clin 30: 157-164, 2020.

31. Kyriakis JM and Avruch J: Protein kinase cascades activated by stress and inflammatory cytokines. BioEssays 18: 567-577, 1996.

32. Soriani A, Borrelli C, Ricci B, Molfetta R, Zingoni A, Fionda C, Carnevale S, Abruzzese MP, Petrucci MT, Ricciardi MR, et al: p38 MAPK differentially controls NK activating ligands at transcriptional and post-transcriptional level on multiple myeloma cells. OncoImmunology 6: e1264564, 2016.

33. Chen XH, Lu LL, Ke HP, Liu ZC, Wang HF, Wei W, Qi YF, Wang HS, Cai SH and Du J: The TGF- $\beta$-induced up-regulation of NKG2DLs requires AKT/GSK-3 $\beta$-mediated stabilization of SP1. J Cell Mol Med 21: 860-870, 2017.

This work is licensed under a Creative Commons Attribution-NonCommercial-NoDerivatives 4.0 International (CC BY-NC-ND 4.0) License. 